\title{
Self-similar solutions of asymmetric Rayleigh-Taylor mixing
}

\author{
A. Hillier ${ }^{1,}$ a) \\ CEMPS, University of Exeter, Exeter EX4 4QF U.K.
}

(Dated: 2 December 2019)

The late nonlinear phase of the Rayleigh-Taylor instability is characterised by the self-similar expansion of the instability mixing layer given at late times by $h \approx \alpha A g t^{2}$. In this paper we present a new model of this mixing layer, based on a piecewise step function approximation where the main constraint imposed is conservation of mass. This model is used to predict the structuring of the mean density of the layer and the asymmetry of the layer for a given Atwood number. By comparing to experimental data and simulation results we confirmed the predictions of the model for the asymmetry of the $\alpha$ values. Our model leads to a simple correction to the formulation of the expansion of the mixing layer which is consistent with an $\alpha$ for a given system that is independent of the density difference for both immiscible fluids and miscible fluids with low mass diffusion. As the model predicts the mean density profile, it can be used to state the energy released by the instability.

\section{INTRODUCTION}

The Rayleigh-Taylor instability (RTi) ${ }^{1,2}$ occurs when a heavy fluid is supported above a light fluid against gravity or a light fluid is accelerated into a heavy fluid. This instability plays an important role in many systems including Supernova mixing ${ }^{3}$, inertial confinement fusion ${ }^{4}$ and solar prominences plume formation ${ }^{5,6}$ making the nonlinear development of the instability an important topic of research.

As the initial instability develops, nonlinearities form, then after further development in this nonlinear regime the initial conditions are forgotten by the system and the width of the Rayleigh-Taylor mixing layer evolves in a self-similar fashion. The first model of the self-similar evolution, based on dimensional analysis, of this mixing ${ }^{7}$ predicts the mixing to happen such that the height and depth of the mixing layer evolves as:

$$
h=\alpha A g t^{2},
$$

where $A=\left(\rho_{2}-\rho_{1}\right) /\left(\rho_{2}+\rho_{1}\right)$ is the Atwood number calculated from the lower (subscript 1) and upper (subscript 2) densities, $g$ is gravitational acceleration and $\alpha$ is the nonlinear growth rate of the layer. Analysis of the NavierStokes equation ${ }^{8}$ shows that the self-similar evolution of the Rayleigh-Taylor mixing results in the following ODE for the mixing layer width,

$$
\dot{h}^{2}=4 \alpha g A h
$$

Equation (2) has the solution

$$
h=\alpha A g t^{2}+2\left(\alpha A g h_{0}\right)^{1 / 2} t+h_{0},
$$

where $h_{0}$ is the height at which the self-similar evolution begins. At late times this just reduces the model that comes from dimensional analysis ${ }^{7}$. As the late time limit is hard to achieve ${ }^{9}$, calculations of $\alpha$ using this method can depend heavily on the determination of the virtual origin, i.e. calculation $h_{0}$.

\footnotetext{
a)Electronic mail: a.s.hillier@exeter.ac.uk
}

The value of $\alpha$ has been the subject of much study ${ }^{9-13}$. In general, the equations presented in the previous paragraph are used to determine its value, but there are a range of methods that have been applied in the literature ${ }^{12}$. Values of $\sim 0.05$ found in experiments ${ }^{12,14}$, but values of 0.027 are found in 3D numerical simulations ${ }^{9,12,15,16}$. The initial spectrum of the perturbations can have a huge influence on the value of $\alpha$ obtained, with a difference of a factor of 2 to $3^{11,17}$ as a result of the initial spectrum. This can potentially explaining the difference between experiments and simulations.

Dimonte and Schneider ${ }^{14}$ experimentally investigated the difference between the measured $\alpha$ values in immiscible fluids for the rising bubbles and falling spikes across a range of Atwood numbers finding an increase in the $\alpha$ value calculated for downward falling spikes $\left(\alpha_{\mathrm{s}}\right)$ as the Atwood number increases. In miscible fluids, various levels of asymmetry between $\alpha_{\mathrm{b}}$ and $\alpha_{\mathrm{s}}$ (or the height $h_{\mathrm{b}}$ and depth $h_{\mathrm{s}}$ of the mixing layer) have been found in the mixing layer. In some cases ${ }^{18}$ the asymmetry was found to be similar to that of the immiscible case, but in other investigations ${ }^{15,16,19,20}$ the asymmetry of miscible fluids at large $A$ was found to much less than that of immiscible fluids. This difference may be attributed to the importance of mass diffusion in different cases. For an overall review, see Zhou et al. ${ }^{9}$.

The asymmetry in $\alpha$, and what this implies for the density distribution of the layer, represents a significant uncertainty in the determination of the value for $\alpha$. However, currently only empirical models based on fits to simulations ${ }^{19}$ or experimental data ${ }^{14}$ exist. In this paper we proposes an extension to the late time model, $h=\alpha A g t^{2}$, based on a hierarchy of selfsimilar solutions that is applicable to immiscible fluids and miscible fluids with small mass diffusion.

This model takes into account the asymmetries associated with large Atwood numbers and can predict the density distribution that result from these asymmetries.

\section{MIXING BY THE RAYLEIGH-TAYLOR INSTABILITY AS A HIERARCHY OF SELF-SIMILAR SOLUTIONS}

The fundamental situation we will study is for when two layers (of densities $\rho_{1}$ and $\rho_{2}$ with the plane of the density 
discontinuity at $y=0$ ) mix by the late-time behaviour of the nonlinear evolution of the RTi. The model we propose to capture the mean-field evolution of the mixing layer is based on two simple premises. Firstly that mass is conserved and secondly that any mixing layer is placed so that it releases the most gravitational potential energy. Following these rules, we create a mixing layer between the two constant density layers of our initial conditions, where here a mixing layer is modelled by a layer of constant average density (averaged in the directions perpendicular to the acceleration) of width $l$.

To determine the layer position which maximises the release of gravitational potential energy (GPE) in the layer is a simple exercise of placing the centre of the layer between $y / l=[-1 / 2,1 / 2]$. The initial GPE, calculated using $\mathrm{E}_{\mathrm{GPE}}=m g h$ with $m$ the mass of the layer, $g$ the magnitude of the constant gravity, and $h$ the height of the centre of mass from the position of discontinuity, is given by:

$$
\mathrm{E}_{\mathrm{GPE}-\mathrm{INIT}}=\frac{g l^{2}}{2}\left[\left(\frac{1}{2}+\frac{y}{l}\right)^{2} \rho_{2}-\left(\frac{1}{2}-\frac{y}{l}\right)^{2} \rho_{1}\right] .
$$

In the new layer formed by mixing, the mass is given by

$$
m_{\operatorname{mix}}=l\left[\left(\frac{1}{2}-\frac{y}{l}\right) \rho_{1}+\left(\frac{1}{2}+\frac{y}{l}\right) \rho_{2}\right] \text {. }
$$

and with the centre of mass of the layer at $y / l$ this gives a GPE of

$$
\mathrm{E}_{\mathrm{GPE}-\mathrm{MIX}}=g l^{2} \frac{y}{l}\left[\left(\frac{1}{2}+\frac{y}{l}\right) \rho_{2}+\left(\frac{1}{2}-\frac{y}{l}\right) \rho_{1}\right],
$$

We can therefore define the change in GPE $\left(\Delta E_{\mathrm{GPE}}\right)$ to be given by

$$
\begin{aligned}
\Delta E_{\mathrm{GPE}} & =\mathrm{E}_{\mathrm{GPE}-\mathrm{MIX}}-\mathrm{E}_{\mathrm{GPE}-\mathrm{INIT}} \\
& =\frac{g l^{2}}{2}\left(\frac{1}{4}-\frac{y^{2}}{l^{2}}\right)\left(\rho_{1}-\rho_{2}\right) .
\end{aligned}
$$

This has two important properties: 1) the distribution is symmetric around $y=0$ and 2) it is negative (there has been energy released) if $\rho_{2}>\rho_{1}$. As the value of $\Delta E_{\mathrm{GPE}}$ must be zero at $y= \pm 1 / 2$, then for $\rho_{2}>\rho_{1}$ this gives $y=0$ is a minimum and this position for the layer placement releases the most energy.

Figure 1 shows the energy release normalised so the minimum value is -1 and highlights that the most appropriate position of the layer is that it is centred at the position of the original discontinuity. Therefore, conservation of mass demands the constant average density value is given by the arithmetic mean of the two layers. In the late time RTi mixing, the lower and upper edges of this layer as determined by the depths and heights of the spikes and bubbles respectively are given by $h_{\mathrm{s}, \mathrm{b}}= \pm \alpha_{\mathrm{u}} A g t^{2} / 2$, where $A$ is the Atwood number and $\alpha_{\mathrm{u}}$ is a constant of the mixing. Note that the factor of $1 / 2$ appears in the equation for $h_{\mathrm{s}, \mathrm{b}}$ to signify that a layer centered on the position of the discontinuity is the placement that releases most energy.

This average density profile now results in two density jumps (both half the density difference of the original jump)

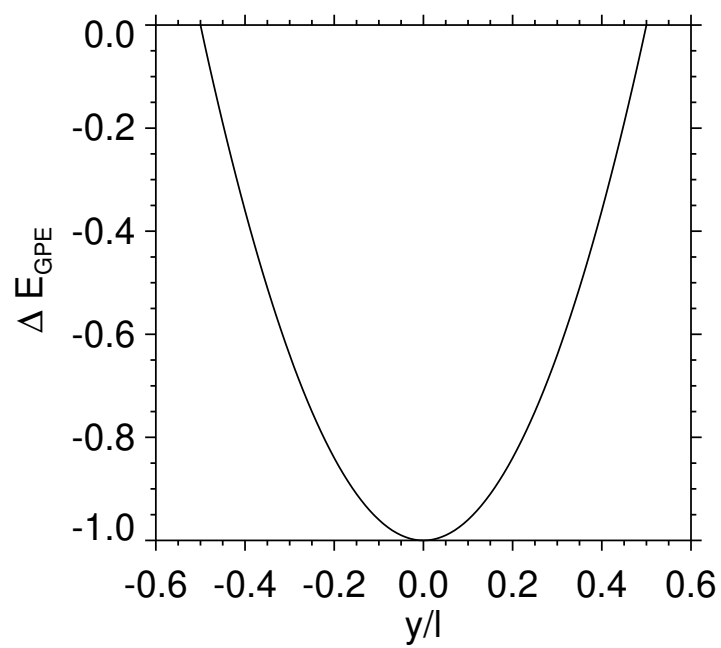

FIG. 1. Calculation of the difference in the GPE between the new layer created by mixing and the original density discontinuity $\left(\Delta E_{\mathrm{GPE}}\right)$ for a mixing layer of width $l$ against the normalised position of the centre of the layer $(y / l)$. The energy difference has been normalised such that the largest difference has a value of -1 .

that form between the mixing layer and the low density region at one end and with the mixing layer and the high density region at the other, and two local Atwood numbers associated with these jumps. These two layers mix in the same self-similar fashion as the first layer over the same time scale meaning these new mixing layer heights are given by $h_{\mathrm{s}, \mathrm{b}}= \pm \alpha_{\mathrm{u}} A_{\text {local }} g t^{2} / 2$, where $A_{\text {local }}$ is the local Atwood number for each mixing layer. This creates four density jumps of one quarter the original density difference all characterised by four local Atwood numbers. This process continues until the jumps becomes infinitesimally small, i.e. a continuous density profile has been achieved.

At each layer, the thickness of that mixed region is determined by the same self similar model as given by Equation 1. We propose that the values of $\alpha_{u}$ (a universal mixing constant), $t$ and $g$ are the same at each layer of the hierarchy which implies that the only parameter that determines the relative thickness of a given layer is the local Atwood number. For the height of the $m^{\text {th }}$ step of the $n^{\text {th }}$ layer of the hierarchy from its bottom to its top we have:

$$
H_{\mathrm{n}, \mathrm{m}}=\alpha_{u} A_{\mathrm{n}, \mathrm{m}} g t^{2}
$$

which will be $A_{\mathrm{n}, \mathrm{m}} / A_{1,1}$ smaller than the thickness of the first level of the mixing layer, where $A_{\mathrm{n}, \mathrm{m}}$ is the local Atwood number associated with the mixing at that point in the hierarchy, and $A_{1,1}=A$. It is worth noting that the maximum value of $m$ for a given $n$ (and with it the number of layers being mixed at that level) is given by $2^{n-1}$.

Figure 2 shows the development of the hierarchy of self similar solutions for two different density jumps $\left(\Delta \rho / \rho_{1}=16\right.$ on the top and $\Delta \rho / \rho_{1}=0.1$ on the bottom). This is shown up to the $n=3$ level. The black lines show the density distribu- 

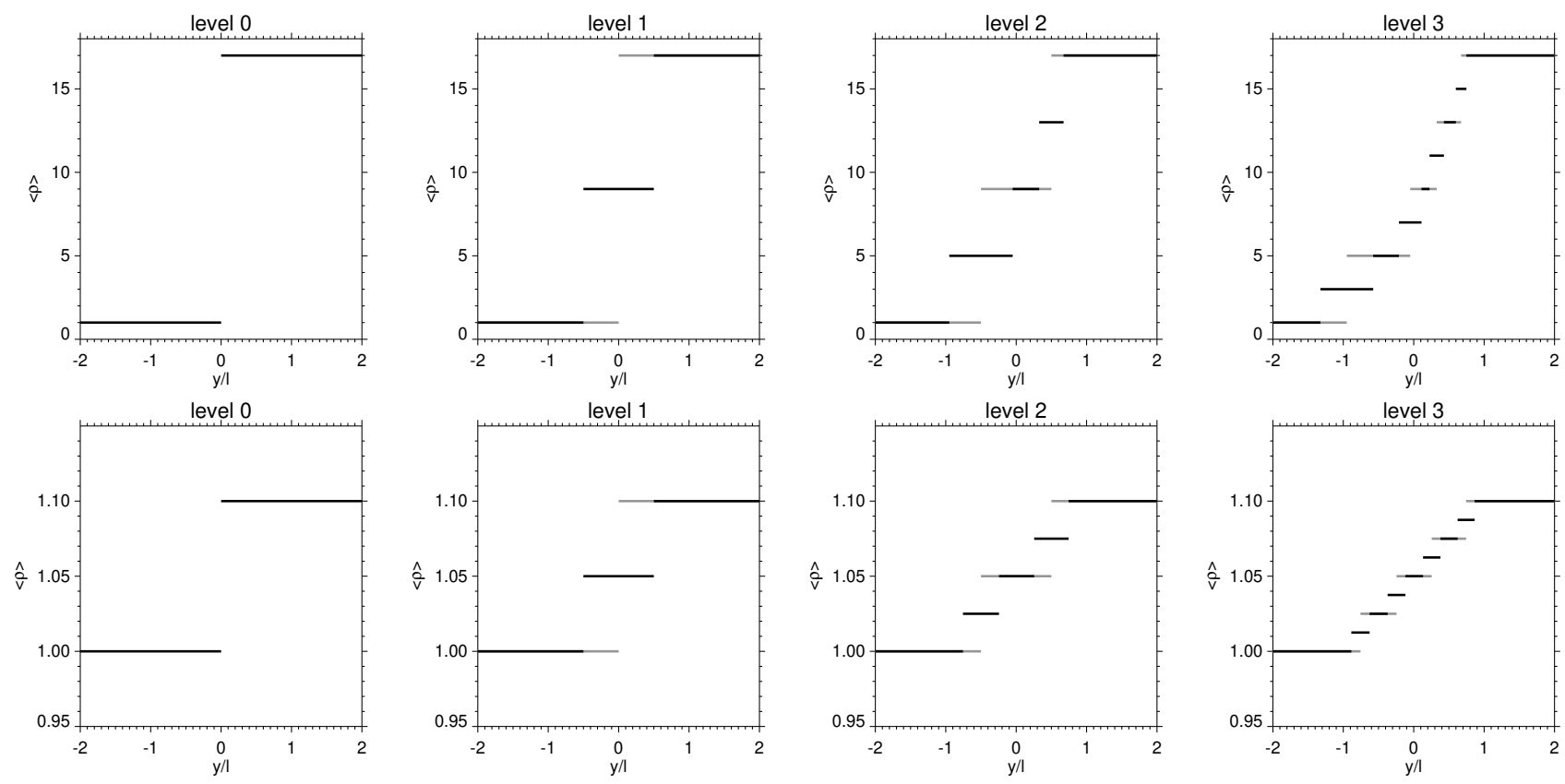

FIG. 2. Up to the $n=3$ level of the hierarchy for a difference of $\Delta \rho / \rho_{1}=16$ (top) and $\Delta \rho / \rho_{1}=0.1$ (bottom). At each level of the hierarchy, the density jumps from the previous layer are mixed. After constructing the $\mathrm{n}^{\text {th }}$ level there are $2^{n}$ surfaces to mix at the next level. The grey lines show the regions of previous layers that have been eroded by the new mixing layer.

tion and the grey lines show the regions of previous levels that are eroded away by the new level of mixing. It can be easily seen from the figure that the large density jump results in a very asymmetric density distribution, but the small density jump gives a linear profile. In the following subsections we will describe mathematically why different Atwood numbers result in different heights, depths and density profiles of the mixing region.

\section{A. The upper and lower edge of the mixing layer}

The position of the upper edge of the mixing layer, the height often denoted $h_{\mathrm{b}}$, and the lower edge of the mixing layer, the depth often denoted $h_{\mathrm{s}}$, are given as the sum of the half widths of the various layers of which they are composed. For any density jump this is given by:

$$
\begin{aligned}
h_{\mathrm{s}}= & -\frac{1}{2} \alpha_{u} g t^{2} \sum_{n=1}^{n=\infty} A_{n, 1}=-\frac{1}{2} \alpha_{u} g t^{2} A \sum_{n=1}^{n=\infty} A^{-1} \frac{\Delta \rho / 2^{n-1}}{2 \rho_{1}+\Delta \rho / 2^{n-1}} \\
& =-\frac{1}{2} \alpha_{u} g t^{2} A \sum_{n=1}^{n=\infty} \frac{1}{2^{n-1}(1-A)+A} \\
h_{\mathrm{b}}= & \frac{1}{2} \alpha_{u} g t^{2} \sum_{n=1}^{n=\infty} A_{n, 2^{n-1}}=\frac{1}{2} \alpha_{u} g t^{2} A \sum_{n=1}^{n=\infty} A^{-1} \frac{\Delta \rho / 2^{n-1}}{2 \rho_{2}-\Delta \rho / 2^{n-1}} \\
& =\frac{1}{2} \alpha_{u} g t^{2} A \sum_{n=1}^{n=\infty} \frac{1}{2^{n-1}(1+A)-A}
\end{aligned}
$$

\section{Small density difference limit}

In the limit $A \ll 1$ the heights of the left most and right most $\mathrm{n}^{\text {th }}$ level mixed layer are given by:

$$
\begin{aligned}
& H_{\mathrm{n}, \mathrm{L}}=\alpha_{u} A_{\mathrm{n}, 1} g t^{2}=\alpha_{u} \frac{A}{2^{n-1}(1-A)+A} g t^{2} \approx \alpha_{u} \frac{A}{2^{n-1}} g t^{2} \\
& H_{\mathrm{n}, \mathrm{R}}=\alpha_{u} A_{\mathrm{n}, \mathrm{r}} g t^{2}=\alpha_{u} \frac{A}{2^{n-1}(1+A)-A} g t^{2} \approx \alpha_{u} \frac{A}{2^{n-1}} g t^{2} .
\end{aligned}
$$

Therefore the position of the edges of the mixed layer is given by:

$$
\begin{aligned}
& h_{\mathrm{s}} \approx \frac{1}{2} \alpha_{u} g t^{2} A \sum_{n=1}^{n=\infty} \frac{1}{2^{n-1}}=\alpha_{\mathrm{u}} g t^{2} A \sum_{n=1}^{n=\infty} \frac{1}{2^{n}}=\alpha_{\mathrm{u}} A g t^{2} \\
& h_{\mathrm{b}} \approx \frac{1}{2} \alpha_{\mathrm{u}} g t^{2} A \sum_{n=1}^{n=\infty} \frac{1}{2^{n-1}}=\alpha_{\mathrm{u}} g t^{2} A \sum_{n=1}^{n=\infty} \frac{1}{2^{n}}=\alpha_{\mathrm{u}} A g t^{2}
\end{aligned}
$$

i.e. the sum used to determine the position of the top and bottom has become approximately a geometric series with common factor $1 / 2$. and initial value $\alpha_{\mathrm{u}} A g t^{2} / 2$. Note that only half of the width of each layer is used to determine the distance of the edge of the mixing region from the midplane. In this limit, the height and the depth of the mixing has the same value and we can expect that the density profile will be linearly changing between the two density values over a region of $y= \pm \alpha_{\mathrm{u}} A g t^{2}$. 


\section{Large density difference limit}

In the large density limit, where $A \sim 1$, there is no longer a symmetric mixing layer. Figure 2 shows this for the $\Delta \rho / \rho_{1}=$ 16 case $(A=16 / 18)$, especially that a long tail forms at the lower end of the mixing region. This happens because even though for each new level the difference in density between $\rho_{1}$ and the lower layer is reduced by a factor of two, for initially large Atwood numbers it takes a number of levels before the local Atwood number is significantly smaller than the initial Atwood number. As such, the new layers still add significantly to the thickness of the mixing layer. Conversely, for the layers closest to $\rho_{2}$ they converge very quickly, resulting in the asymmetry. Equations 9 and 10 can be used to calculate the extent of the mixing region.

In the most extreme case, we can have $\rho_{1}=0$. Studying this limit leads to two important bounds on the lower limit of the height of the mixing layer and the upper limit of the depth of the mixing layer. For the lower bound, as $\rho_{1}=0$ the Atwood number at a given level $A_{n, 1}$ will always equal unity. Therefore, the width of the bottom-most layer added to the mixing region at the $n^{\text {th }}$ level will equal that of the layer added at the first level. This results in the depth of the mixing layer becoming infinite. An inifintely fast expansion is obviously an unphysical solution, and shows the limits of this model as $A \rightarrow 1$, which could imply that a self-similar solution is never achieved in this situation. As it is to be expected that $h_{\mathrm{s}}$ is limited by freefall ${ }^{21}$ at $h_{\mathrm{s}}=g t^{2} / 2$, care must be taken in applying this model in situations where the Atwood number is so large that the predicted $h_{\mathrm{s}}$ is larger than this limit. However, it should be noted that the amount of mass in the model that has extended beyond this unphysical point is expected to be very small.

The height of the upper layer is given by the following sum:

$$
h_{\mathrm{b}}=\frac{1}{2} \alpha_{u} g t^{2} \sum_{n=1}^{n=\infty} \frac{1}{2^{n}-1 .} \approx \frac{1.607}{2} \alpha_{u} g t^{2} .
$$

In this case the prefactor becomes $\sim 1.607 / 2$ instead of 1 for the small density limit case, highlighting the expected range in the height of the mixing layer. This does mean that even when comparing the measured value $\alpha_{\mathrm{b}}$ between low and high density difference experiments or simulations care should be taken.

\section{Atwood number dependence}

Now we turn our attention to the predictions of our model across the spectrum of global Atwood numbers. Figure 3 shows the values of $\alpha_{\mathrm{s}}$ (solid line) and $\alpha_{\mathrm{b}}$ (dashed line) normalised by $\alpha_{\mathrm{u}}$ of the mixing layer at different Atwood numbers. Our method for calculating $\alpha_{\mathrm{b}}$ and $\alpha_{\mathrm{s}}$ is:

$$
\begin{aligned}
& \alpha_{\mathrm{s}}=\frac{1}{2} \alpha_{u} \sum_{n=1}^{n=\infty} \frac{1}{2^{n-1}(1-A)+A}, \\
& \alpha_{\mathrm{b}}=\frac{1}{2} \alpha_{u} \sum_{n=1}^{n=\infty} \frac{1}{2^{n-1}(1+A)-A} .
\end{aligned}
$$

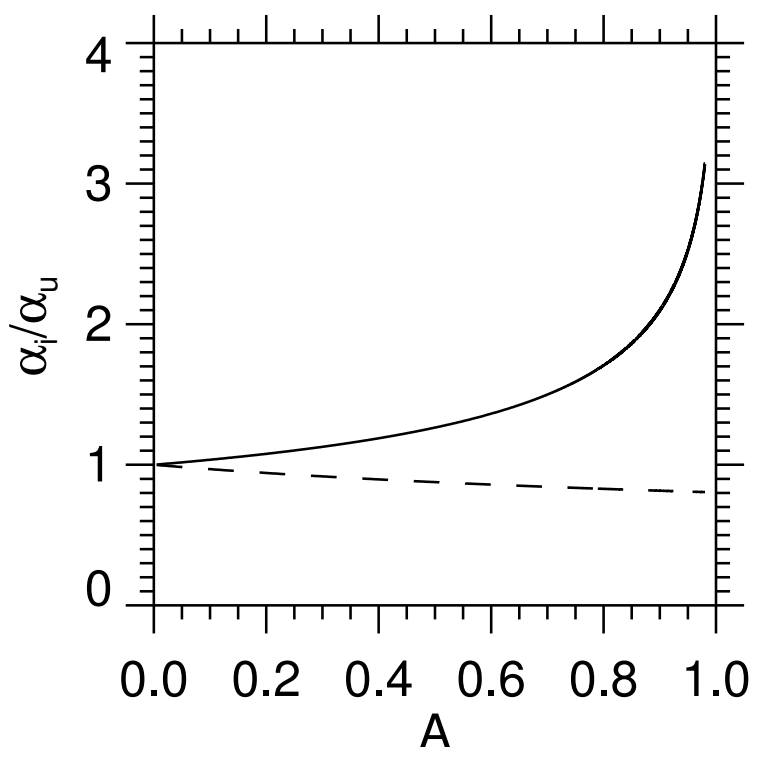

FIG. 3. Ratio of the value of $\alpha_{\mathrm{b}} / \alpha_{u}$ and $\alpha_{\mathrm{s}} / \alpha_{u}$ (bubbles shown in dashed line and spikes in solid line) against global Atwood number.

The weak dependence of $\alpha_{\mathrm{b}} / \alpha_{\mathrm{u}}$ on the Atwood number and the strong dependence of $\alpha_{\mathrm{s}} / \alpha_{\mathrm{u}}$ on the Atwood number are clear.

Figure 4 shows the predicted values of $\alpha_{\mathrm{s}}$ and $\alpha_{\mathrm{b}}$ for $\alpha_{\mathrm{u}}=$ 0.055 and $\alpha_{\mathrm{u}}=0.065$ compared to the experimental results of $\alpha_{\mathrm{s}}$ (open circles ${ }^{14}$ and diamonds ${ }^{18}$ ) and $\alpha_{\mathrm{b}}$ (filled circles ${ }^{14}$ and $\operatorname{crosses}^{18}$ ). By setting a range of $\alpha_{\mathrm{u}}=0.055$ to 0.065 , chosen because of the clustering around these values at small Atwood numbers, it was possible to take these values and use our model to make a prediction of the change in the measured value of $\alpha$ expected in these experiments. As can be see from the figure, the similarity between the experimental results and the model is striking. This is not just for predicting the huge increase in $\alpha_{\mathrm{s}}$ with $A$, but also the gentle decrease of $\alpha_{\mathrm{b}}$ that also appears to exists. Clearly our model represents an important improvement in the measurement of $\alpha$ as it captures the Atwood number related spread and asymmetry. Note that both immiscible $^{14}$ and miscible ${ }^{18}$ experimental results are shown on this plot. The good correlation in behaviour between the two sets of results likely implies that mass diffusion was not important in instability development in the miscible experiments.

\section{B. Structure of the mixing layer}

Another important result of the model is that it predicts the horizontally averaged density distribution $(\langle\rho(y)\rangle)$ of the mixing layer. Though the distribution is required to be monotonic, as can be seen in Figure 2, the more layers added into the hierarchy, the greater complexity that can be seen in the predicted density distribution. In the remainder of this section we will investigate some qualities of the density distribution 


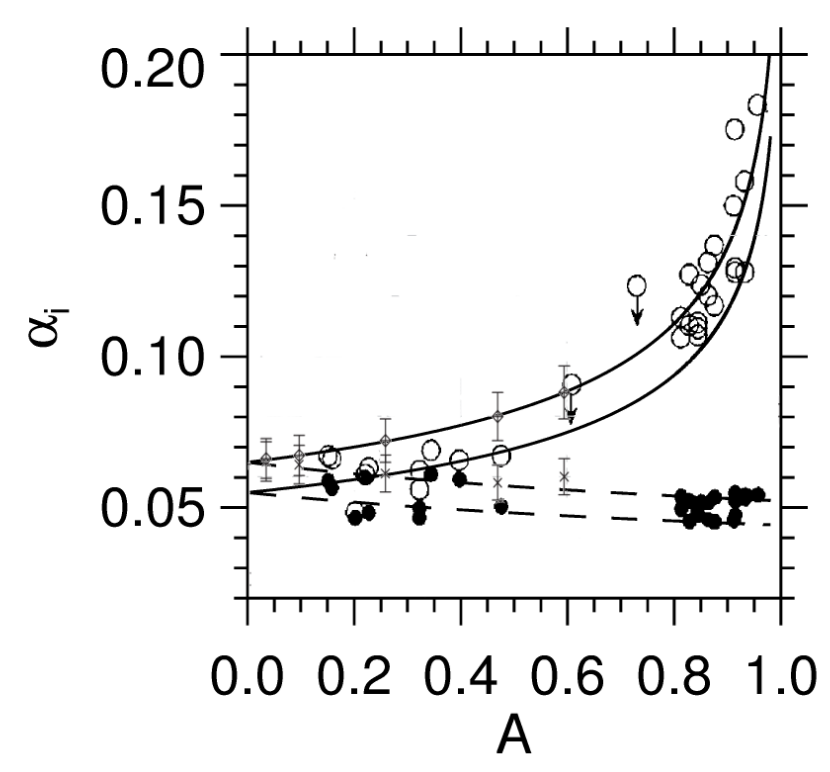

FIG. 4. Experimental results for $\alpha_{\mathrm{b}}$ (filled circles ${ }^{14}$ and diamonds ${ }^{18}$ ) and $\alpha_{\mathrm{s}}$ (empty circles ${ }^{14}$ and crosses $^{18}$ ) as a function of global Atwood number with the theoretical curves for these values using both $\alpha_{\mathrm{u}}=0.055$ and $\alpha_{\mathrm{u}}=0.065$. Diamonds and crosses are taken from Banerjee, Kraft, and Andrews ${ }^{18}$, Journal of Fluid Mechanics, 659, 127-190, reproduced with permission.

our model predicts, and provide a polynomial approximation of the model.

\section{1. $\langle\rho\rangle$ gradient near the edges of mixing layer}

To estimate the gradient at either edge of the mixing layer, we need to see the rate at which the density converges to either $\rho_{1}$ or $\rho_{2}$ with $n$ compared to the rate at which the width of the mixing layer is increased by the $n^{\text {th }}$ mixing layer. For the leftmost or rightmost of the nth levels the density is given as:

$$
\rho_{n, L}=\rho_{1}+\frac{\Delta \rho}{2^{n}}, \quad \rho_{n, R}=\rho_{2}-\frac{\Delta \rho}{2^{n}}
$$

Assuming this density is characteristic of the centre position of the layer, the distance between the $n^{\text {th }}$ and the $n+1^{\text {th }}$ density values is:

$\Delta h_{\mathrm{n}, \mathrm{L}}=\frac{1}{2} A^{-1} \frac{\Delta \rho / 2^{n-1}}{2 \rho_{1}+\Delta \rho / 2^{n-1}}, \quad \Delta h_{\mathrm{n}, \mathrm{R}}=\frac{1}{2} A^{-1} \frac{\Delta \rho / 2^{n-1}}{2 \rho_{2}-\Delta \rho / 2^{n-1}}$

Taking the density difference between the $n^{\text {th }}$ and $n+1^{\text {th }}$ layers divided by the distance gives:

$$
\begin{aligned}
& \frac{\left(\rho_{1}+\Delta \rho / 2^{n+1}\right)-\left(\rho_{1}+\Delta \rho / 2^{n}\right)}{-\Delta h_{\mathrm{n}, \mathrm{L}}}=A\left(\rho_{1}+\frac{\Delta \rho}{2^{n}}\right), \\
& \frac{\left(\rho_{2}-\Delta \rho / 2^{n+1}\right)-\left(\rho_{2}-\Delta \rho / 2^{n}\right)}{\Delta h_{\mathrm{n}, \mathrm{R}}}=A\left(\rho_{2}-\frac{\Delta \rho}{2^{n}}\right) .
\end{aligned}
$$

Taking the limit of $n \rightarrow \infty$, we can calculate the gradient of the density at the edges of the mixing layer, i.e.:

$$
\frac{d\left\langle\rho\left(L_{1}\right)\right\rangle}{d y}=A \rho_{1}, \quad \frac{d\left\langle\rho\left(L_{2}\right)\right\rangle}{d y}=A \rho_{2},
$$

with

$$
L_{1}=\frac{h_{s}}{l}=\frac{1}{2} \sum_{n=1}^{n=\infty} \frac{1}{2^{n-1}(1-A)+A},
$$

and

$$
L_{2}=\frac{h_{b}}{l}=\frac{1}{2} \sum_{n=1}^{n=\infty} \frac{1}{2^{n-1}(1+A)-A},
$$

the nondimensional depth and height of the mixing layer, respectively. We can see that this is the density value multiplied by the global Atwood number. Repeating this process recursively, we find for the $m^{\text {th }}$ derivative:

$$
\frac{d^{m}\left\langle\rho\left(L_{1}\right)\right\rangle}{d y^{m}}=A^{m} \rho_{1}, \quad \frac{d^{m}\left\langle\rho\left(L_{2}\right)\right\rangle}{d y^{m}}=A^{m} \rho_{2} .
$$

\section{Overlapping mixing layers and the existence of a $\langle\rho\rangle$ inflection point}

In this model, as each new set of layers are added the lower levels are eroded. For some of these eroded levels, eventually the higher levels eroding them overshoot and completely remove them. It is necessary to define what happens in this situation.

We propose that once two layers meet, a new mixing layer is formed between them with its width calculated by the local Atwood number and the time remaining $\left(t_{\mathrm{R}}\right)$ until $t$ in the mixing process. Panel (a) of Figure 5 shows the model density distribution at the fourth level (with a approximation of the model from a polynomial distribution - see Section II B 3). In this case the band at $\langle\rho\rangle=9$ has been completely overshot by the layers above and below. Therefore the width of this layer is determined by the process described in the previous paragraph.

The existence of these points mean that the gradient of the average density, when approaching from below, will steepen as this point is approached. Above this point, the gradient will reduce. The implication from our model is that we predict the existence of an inflection point where $\langle\rho\rangle=\rho_{1}+\Delta \rho / 2$.

\section{Approximate functional form of density distribution}

By imposing the appropriate conditions, we investigate if it is possible to approximate the density distribution predicted by the model with a polynomial distribution. Here we look to approximate the density distribution by a k-th order polynomial, i.e.:

$$
\left\langle\rho\left(y^{\prime}\right)\right\rangle \approx \Sigma_{i=0}^{k} A_{i} y^{\prime i}=f\left(y^{\prime}\right)
$$

where $y^{\prime}=y / l$. 
Combining the conditions for the density value and gradient at the two edges of the mixing layer with the condition that the mass in the layer after mixing is the same as the mass before mixing began we can determine the values of the $k+1$ coefficients. Firstly, conservation of mass implies that:

$$
\int_{L 1}^{L_{2}} f\left(y^{\prime}\right) d y=L_{1} \rho_{1}+L_{2} \rho_{2} .
$$

To make sure that the density is continuous, because without this our model implies further mixing will occur until this condition is satisfied, we know that $\left\langle\rho\left(L_{1}\right)\right\rangle=\rho_{1}$ and $\left\langle\rho\left(L_{2}\right)\right\rangle=\rho_{2}$. In Section II B 1 we give the conditions on the derivative, ie. the $m^{\text {th }}$ derivatives of $f$ at $L_{1}$ and $L_{2}$ are:

$$
f^{m}\left(L_{1}\right)=A^{m} \rho_{1}, \quad f^{m}\left(L_{2}\right)=A^{m} \rho_{2}
$$

Note that $L_{1}$ and $L_{2}$ are the normalised (by $\alpha_{\mathrm{u}} A g t^{2}$ ) distances of the two edges of the mixing region from $y^{\prime}=0$. $f\left(y^{\prime}\right)$ can be easily determined by putting these conditions into matrix form and finding the constants.

Figure 5 gives the comparison between the model and the approximate polynomial (taken using $k=8$ ) for the appropriate model parameters. It is clear that for these two density differences (16 with four levels in panel (a) and 0.1 with three levels in panel (b)) that the polynomial is of sufficient order to describe these distributions. To improve the match for larger density distributions, as well as using larger $k$ values, it may be possible to use the inflection point described in Section II B 2.

\section{CONFIRMING THE PREDICTED DENSITY DISTRIBUTION}

In this section, we use comparisons to evaluate the accuracy of the predicted mean density distribution. This is performed through the comparison with both experimental results and those from numerical calculations.

\section{A. Comparison with experimental data}

An important comparison for the density profile solution is with various experimental profiles for the mean density. In this section we will compare the volume fraction of fluid 2, defined as

$$
V F=\frac{\langle\rho\rangle-1 .}{\Delta \rho},
$$

between our prediction of the density distribution in the mixing layer, and some published experiment results. To scale the solution and the experimental data so they have the same $\mathrm{x}$-axis we set that the analytical solution and the experimental data have the same mixing fraction of 0.2 at the same position on the $\mathrm{x}$-axis. No other scaling is performed.

Figure 6 shows a comparison between the analytic solution and the experimental mixing fractions for Atwood numbers of $A=0.2$ and $A=0.32^{14}$. The experimental results (shown in black) and the model (shown in red) match well. For the experiment with $A=0.33$, the $\alpha_{u}$ value that is consistent with the matching of the 0.2 volume fraction is $\alpha_{u}=0.055$. However, the comparison with the $A=0.2$ experiment yields $\alpha_{u}=0.037$. For the case of $\mathrm{A}=0.2$, if we had used the mixing fraction of 0.1 as a reference, the $\alpha_{u}$ value would have been 0.047 , and if we had used 0.8 it would have been 0.044 . These values are roughly consistent with the experimental values measured of $\sim 0.047$ (see Figure 4). Performing the same analysis for the $A=0.33$ case, we find $\alpha_{u}=0.055$ for the mixing fraction of 0.1 , and 0.042 for the mixing fraction of 0.8 . Clearly the 0.8 result is different, but looking at the plot there is a clear hump in the data at this mixing fraction (as there is a clear depression at 0.2 in the $A=0.2$ case) which means that it is a region likely to give an under (over) estimate of $\alpha_{u}$. Note that on top of the issues of fluctuations in the mean density profile, as the method for determining $\alpha_{u}$ used here is based on assuming the virtual origin to be 0 , this introduces an error into this estimate.

Figure 7, shows the volume fraction of fluid 2 distribution for a set of miscible gas mixing experiments ${ }^{18}$ (dot-dashed line) compared with the model (solid red line). Again the $y$ axis has been rescaled so the experimental data and the model volume fraction values are the same at the same $y$ for a volume fraction of 0.2. This comparison has been performed for $A=0.04$, and 0.47. Again, even though these experiments are rather different to those presented in Figure 6, the model has captured the asymmetrical distribution over the big range of Atwood numbers. The match between the experiments and the models was associated with $\alpha_{u}=0.064$, and 0.061 , respectively. Note again that virtual origin effects (i.e. that they are not being taken into account in these estimates) are likely to play a role in the spread of values found here.

\section{B. Comparison with numerical simulations}

To further investigate the applicability of the model to predict the density profile and asymmetry as a result of RTi mixing, we perform 3D hydrodynamic simulations of RayleighTaylor mixing. The basic equations we solve are:

$$
\begin{aligned}
\frac{\partial \rho}{\partial t}+\nabla \cdot(\rho \mathbf{v}) & =0 \\
\frac{\partial}{\partial t}(\rho \mathbf{v})+\nabla \cdot(\rho \mathbf{v} \mathbf{v}+P \mathbf{I}) & =\rho \mathbf{g} \\
\frac{\partial e}{\partial t}+\nabla \cdot[\mathbf{v}(e+P)] & =\rho \mathbf{g} \cdot \mathbf{v}
\end{aligned}
$$

with $g=-0.1, \gamma$ as the ratio of specific heats and set as $5 / 3$, and $e=P /(\gamma-1)+\rho v^{2} / 2$.

These simulations are performed using the (PIP) $\operatorname{code}^{22}$ using a fourth order central difference method. To allow the largest possible inertial range of any turbulent behaviour to develop, we do not include explicit viscosity. However, for stability of the scheme we employ an artificial viscosity/diffusion of the conserved quantities using a min-mod limiter with the diffusion parameter $\theta$ set to 1.8 (with 2 as the least diffusive and 1 as the most) ${ }^{23}$, implying that mass, momentum and energy diffusion are non-zero at the grid scale. 
(a)

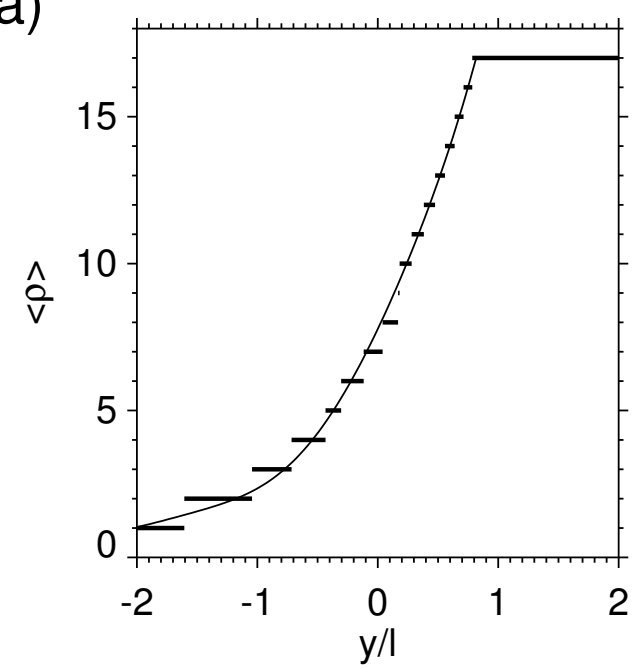

(b)

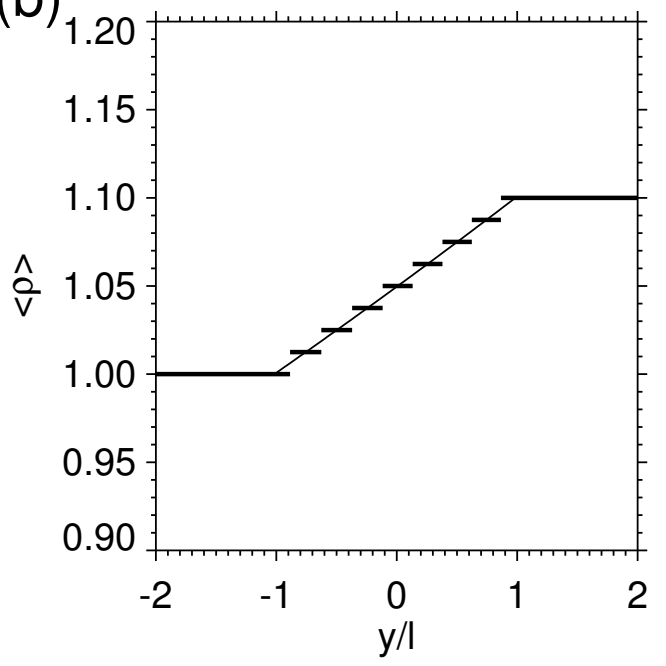

FIG. 5. Comparison of the model with the polynomial approximation for density differences of 16 (panel (a) four levels) and 0.1 (panel (b) three levels). In each panel the thick horizontal lines are the model densities and the continuous line is the distribution given by the polynomial. Note that in panel (a) the bar at $\langle\rho\rangle=9$ is determined by the secondary mixing processes described in Section II B 2.

(a)

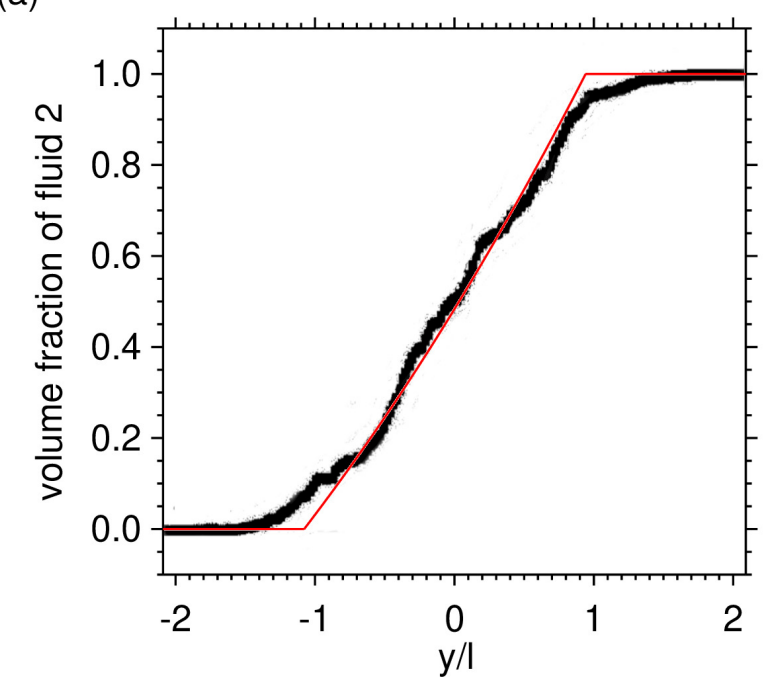

(b)

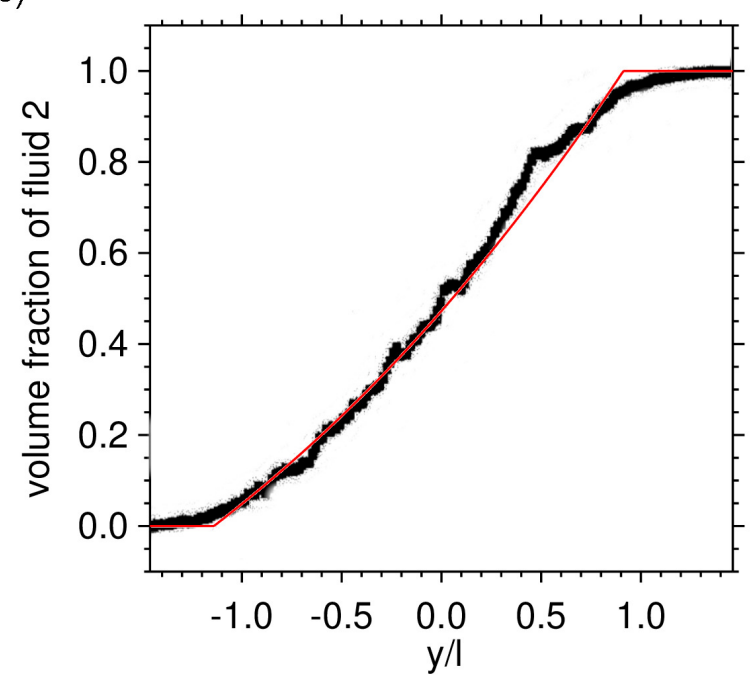

FIG. 6. Experimental mixing fractions for $A=0.2$ (left panel), and $A=0.32$ (right panel) taken from Dimonte and Schneider ${ }^{14}$ shown in black. These are compared with the model prediction for the mixing fraction shown in red.

The initial conditions are:

$$
\rho=\left\{\begin{array}{ll}
\rho_{2} & \text { if } y>0 ; \\
\rho_{1} & \text { if } y<0
\end{array}, \quad V_{x}=V_{z}=0, \quad P=P_{0}+\rho g y,\right.
$$

where $\rho_{1}=1, P_{0}=2$, and $\rho_{2}$ is 1.1 (Figure 8 (a)) and 17 (Figure 8 (b)). The instability is seeded by a random noise perturbation in $V_{y}$ of the order of 1 per cent of sound speed. We use periodic boundary conditions in the side boundaries and reflective at the top and bottom.
The resolution of the simulations is $200 \times 1000 \times 200$ taking the grid size in all directions to be the same. The horizontal domain size is $x=z=[-0.1,0.1]$ and $y=[-0.5,0.5]$ for the $\rho_{2}=1.1$ case. In the $\rho_{2}=17$ case we reduce the range of the horizontal and vertical domains by a factor of 100. It is worthwhile to note that though we have chose our initial conditions to match exactly the initial conditions of the model, the use of a compressible code means that the uniform density regions are not neutrally buoyant ${ }^{24}$ However, as the vertical scale of the simulation compared to the pressure scale 
(a)

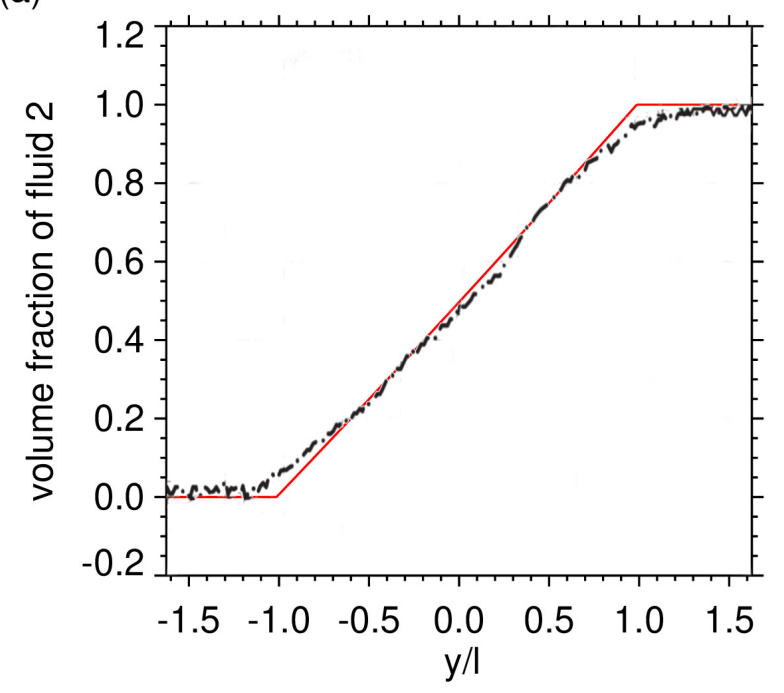

(b)

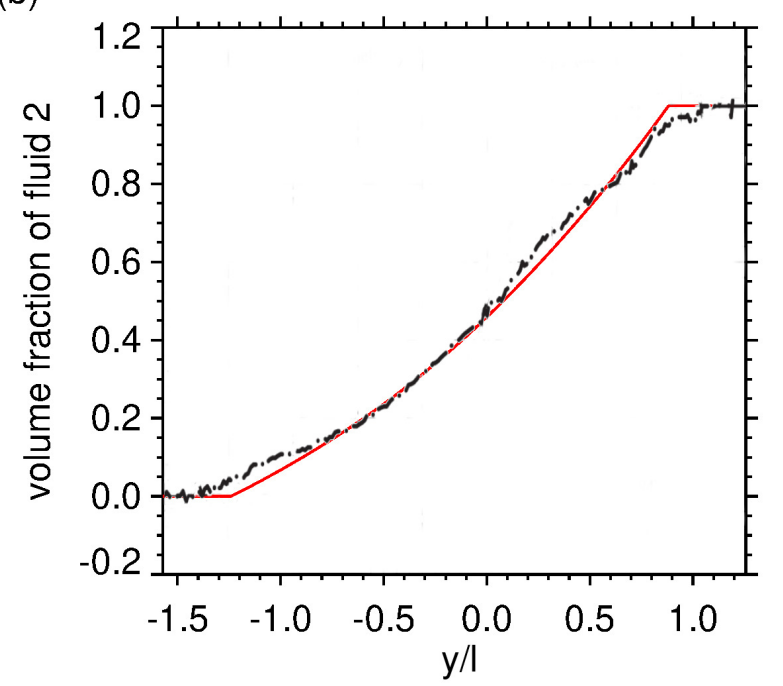

FIG. 7. Comparison between experimental data (dot-dashed line) taken from Banerjee, Kraft, and Andrews ${ }^{18}$, Journal of Fluid Mechanics, $659,127-190$, reproduced with permission, and the model mixing fraction profile (red line) at $A=0.04$ (a) and $A=0.47$ (b)

height is small ( $1 \%$ or less), the timescale for these dynamics is much longer than the simulation timescale and as such will not play an important role in determining the mean density profile. Though these simulations are performed with a compressible code, the simulations are in a regime where the compressible effects are small.

Figure 8 shows a snapshot of the simulations during the later evolution where the nonlinear mixing has developed In this figure panel (a) is from a simulation with $\rho_{2}=1.1$ and panel (b) is from a simulation with $\rho_{2}=17$. The asymmetry that exists in the high density difference simulation compared to the low density difference simulation is clear when comparing panels (a) and (b).

Figure 9 shows two attempts to measure $\alpha_{u}$ from the 3D simulations. One using a revised version of Equation 1 and the other using an equation similar to Equation 2. For the revised version of Equation 1 we have

$$
\alpha_{\mathrm{u}}=\frac{\alpha_{b}}{L_{2}}=\frac{h_{b}}{A g t^{2} L_{2}}=\frac{\alpha_{s}}{L_{1}}=\frac{h_{s}}{A g t^{2} L_{1}} .
$$

Equation 2 can be revised to solve for $\alpha_{\mathrm{u}}$ in the following way

$$
\alpha_{\mathrm{u}}=\frac{\alpha_{b}}{L_{2}}=\frac{\dot{h}_{b}^{2}}{A g h_{b} L_{2}}=\frac{\alpha_{s}}{L_{1}}=\frac{\dot{h}_{s}^{2}}{A g h_{s} L_{1}} .
$$

In fact this can be revised into a general equation for a given value of the mean density $(\langle\rho\rangle)$ in the layer of

$$
\alpha_{\mathrm{u}}=\frac{\dot{h}(\langle\rho\rangle)^{2}}{4 A g h(\langle\rho\rangle) L(\langle\rho\rangle)},
$$

where $L(\langle\rho\rangle)$ is the non-dimension height of that particular value of $\langle\rho\rangle$ in the mixing layer. The estimates of $\alpha_{u}$ made using the data in panel (b) of Figure 9 are shown in table I.
TABLE I. Measured values of $\alpha_{u}$ for the 3D simulations using the results from panel $b$ of Figure 9

\begin{tabular}{cccc}
\hline$\Delta \rho / \rho_{1}$ & measurement point & $\alpha_{u}$ & error \\
\hline 0.1 & top & 0.028 & 0.013 \\
& bottom & 0.025 & 0.011 \\
& whole width & 0.026 & 0.009 \\
16 & top & 0.033 & 0.016 \\
& bottom & 0.021 & 0.010 \\
& whole width & 0.023 & 0.008 \\
\hline
\end{tabular}

These $\alpha_{\mathrm{u}}$ values are calculated at the 3\% tolerance for the low density case and the $10 \%$ tolerance for the high density contrast case.

The results here bare a strong similarity to those of other studies of the mixing parameter ${ }^{16}$ where there is often a big spread in the values obtained and a lot of noise. As such, the errors presented for each measurement are a large proportion of the $\alpha_{u}$ value obtained. As such we look at the comparison of the simulated mean density profile to that of the model for greater confirmation that the model is able to explain the asymmetry of the simulation results.

The two panels Figure 10 show the comparison between the mean density profile and the theoretically predicted density profile for $1 / 21$ (panel a) and $A=16 / 18$ (panel b) respectively. To make this plot, the width of the mixing layer in the mean density is normalised to the width of the predicted layer. This is done by measuring the width of the simulated mixing layer by determining the positions of variations from the background values of $0.01 \Delta \rho$, and normalising the separation between these two values to the width of the model layer at the same tolerance. For the high density difference case, there are some differences between theoretical prediction and 

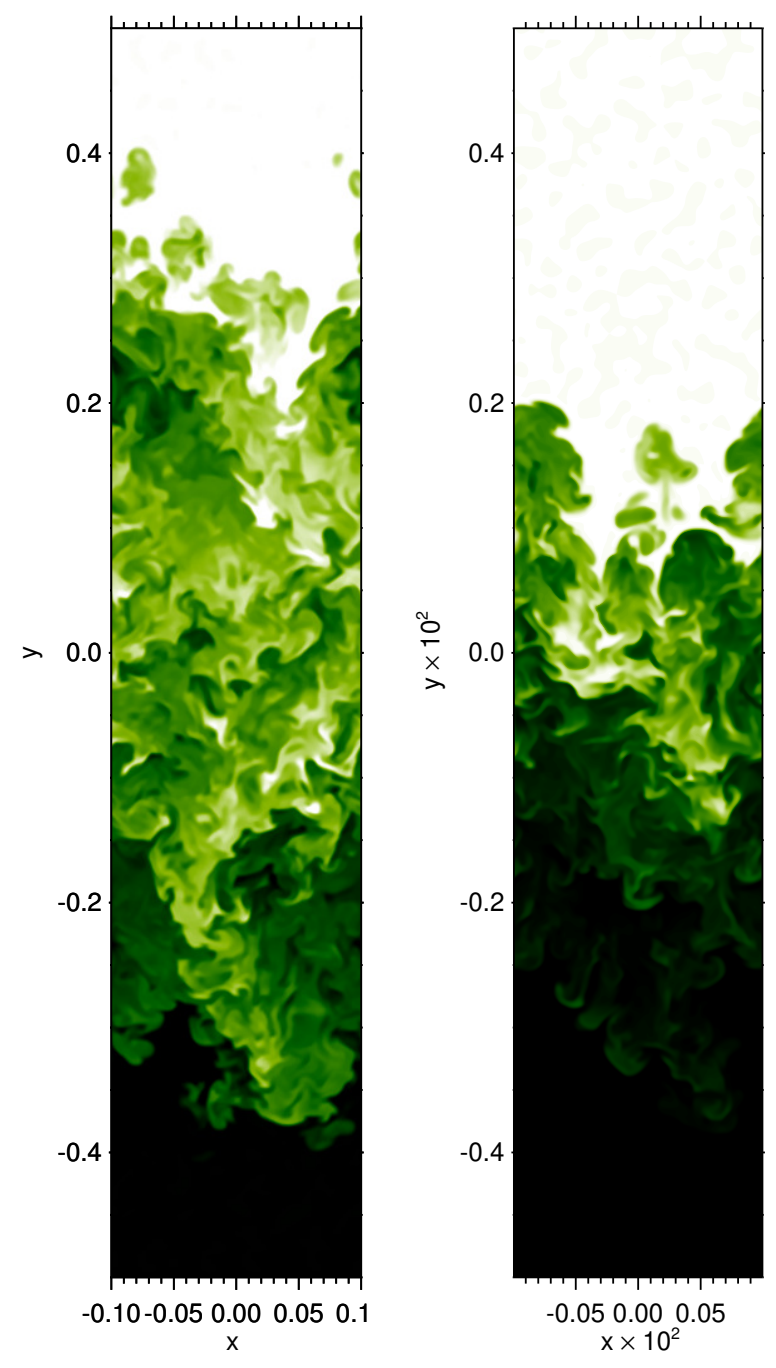

FIG. 8. 2D slice through 3D Simulations of hydrodynamic RT mixing when the density difference is $\Delta \rho / \rho_{1}=0.1$ (a) and $\Delta \rho / \rho_{1}=16$ (b).

the simulations, but the general profile agrees well with the simulation. For the low density difference case the theoretical prediction agrees excellently with the simulated density profile.

\section{Comparison with incompressible RTi simulation results ${ }^{25,26}$}

We have presented a comparison between a set of simulations and the model. However there are a number of simulations in the published literature, some with very high resolution, meaning there is greater opportunity for comparison. Here we make a similar comparison between the model and some simulation data for the mixing fraction as has been applied earlier in the paper to experimental data from one such numerical study ${ }^{25,26}$. The study under comparison ${ }^{25,26}$ is of the incompressible RTi using a grid of $1024^{2} \times 4608$ and simulating the dynamics with the CFDNS code, which uses a mixed spectral- $6^{\text {th }}$ order compact finite differences scheme. These simulations have both explicit viscosity and Fickian mass diffusion.

Figure 11 gives the comparison between the model mixing fraction curve and that found from the simulations using Atwood numbers of $0.04,0.5,0.75$ and 0.9 . The simulation data is shown by the solid black line with the model as the solid red line. As with the previous comparisons with experimental data, the $\mathrm{x}$-axis of the simulation data has been re-normalised so that the 0.2 mixing fraction of both the simulations and the model have the same position. As can be seen from the figure, over a large range of mixing fractions $(\sim 0.1$ to 0.8$)$ the model captures the mean density distribution found in the simulations. The most notable departures between the model and the simulation data are seen at the mixing fractions close to unity where the simulation presents more diffuse structures than those predicted by the model.

It is this upper region where we could expect that the mass diffusion is most effective, because this is where the second derivative of the mean density is largest. From the model presented in the is paper the second derivative of the mean density would be infinite at the point where the two layers join and just inside the mixing region where we predict the second derivative to be

$$
\frac{d^{2}\left\langle\rho\left(L_{2}\right)\right\rangle}{d y^{2}}=A^{2} \rho_{2} .
$$

At earlier times in the self-similar evolution, the increased diffusion in this region is likely to spread out the mixing layer around this point. However, at much later times, the size of the diffusive region compared to the mixing region should shrink (scaling approximately as $t^{-3 / 2}$ ) which can be expected to increase the accuracy of the model at these times. It would be interesting to perform a set of simulations to confirm this hypothesis.

\section{ENERGY AVAILABLE FOR TURBULENT FLOWS}

The development of the nonlinear RTi by its very nature is releasing gravitational potential energy from the initial density distribution and using this to drive flows. Due to the generally chaotic nature of the system, given a large enough Reynolds number the flows will become turbulent ${ }^{15}$. To investigate this turbulence, high-resolution 3D numerical simulations are generally necessary. However, the model we propose allows the determination of the energy extracted from the background density profile, and with this an upper bound for the turbulent energy can be determined.

Figure 12 (panel a) shows the energy release for different $A$. Naturally the greater energy release occurs for larger $A$ values. However, when this value is normalised by the energy release in the first layer of the expansion (panel b) then there is only a growth of $\sim 20 \%$ trend with $A$. Therefore, larger $A$ releases more energy, but it is not significantly more efficient at releasing energy than the smaller $A$ situations. 
(a)

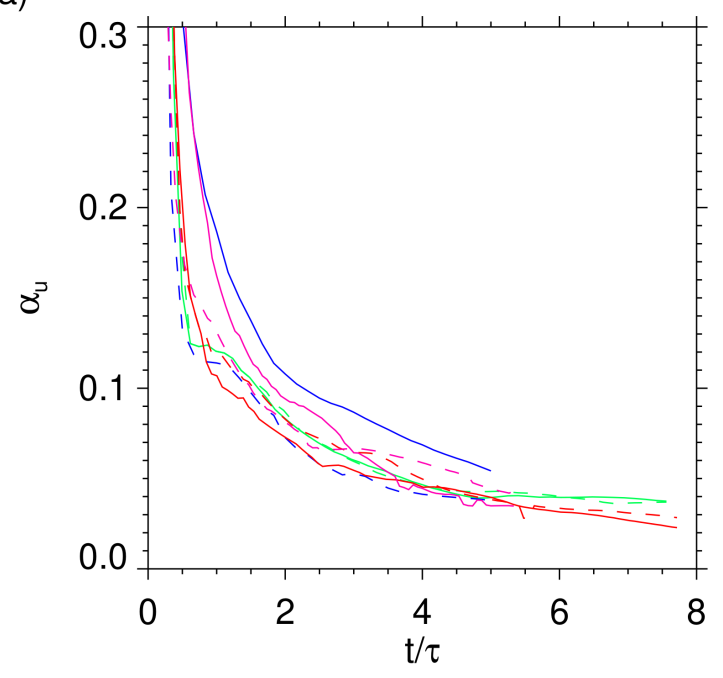

(b)

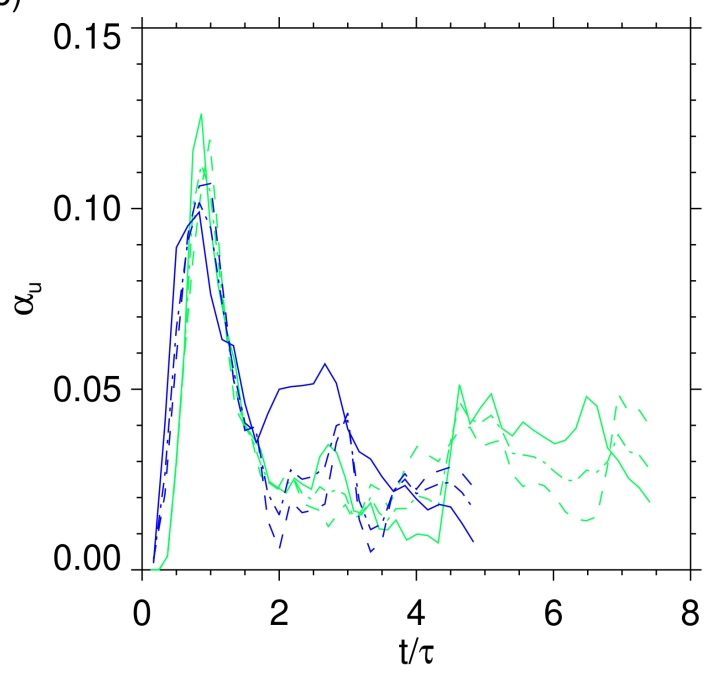

FIG. 9. Panel (a) shows the calculated value of $\alpha_{u}$ in the simulations as calculated from $h_{s} /\left(A g t^{2} L_{1}\right)$ for the spikes (dashed lines) and $h_{b} /\left(A g t^{2} L_{2}\right)$ for the bubbles (solid lines) for the 3D simulations $(A=16 / 18$ in blue and $A=1 / 21$ in green) and for reference $2 \mathrm{D}$ simulations of the same horizontal and vertical resolution as their 3D counterparts $(A=16 / 18$ in pink and $A=1 / 21$ in red). The simulations appear to be converging on a value of $\alpha_{u}$ of $\alpha_{u} \approx 0.025$. Note that for the large $A$ simulations the bubbles and spikes converge to the $\alpha_{u}$ value at different rates. Panel (b) uses the $\dot{h_{s}}{ }^{2} /\left(4 \alpha g A h_{s} L_{1}\right)$ measure for the spikes and $\dot{h_{b}}{ }^{2} /\left(4 \alpha g A h_{b} L_{2}\right)$ for the bubbles to determine $\alpha_{u}$. Colours and line types have the same meaning as panel (a). There has been the addition of the dash-dot line which uses the full width of the layer to estimate $\alpha_{u}$. Though this plot is quite noisy, the average values found for $\alpha_{\mathrm{u}}$ are close to 0.025 (see table I). Here $\tau$ is given by $\tau=\sqrt{g /\left(W_{x} A\right)}$ with $W_{x}$ the size of the domain in the $x$ direction.

(a)

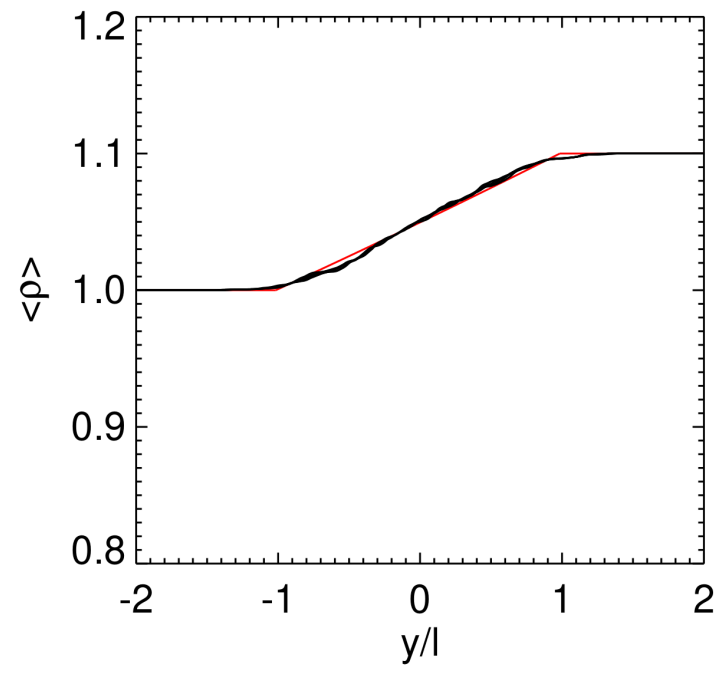

(b)

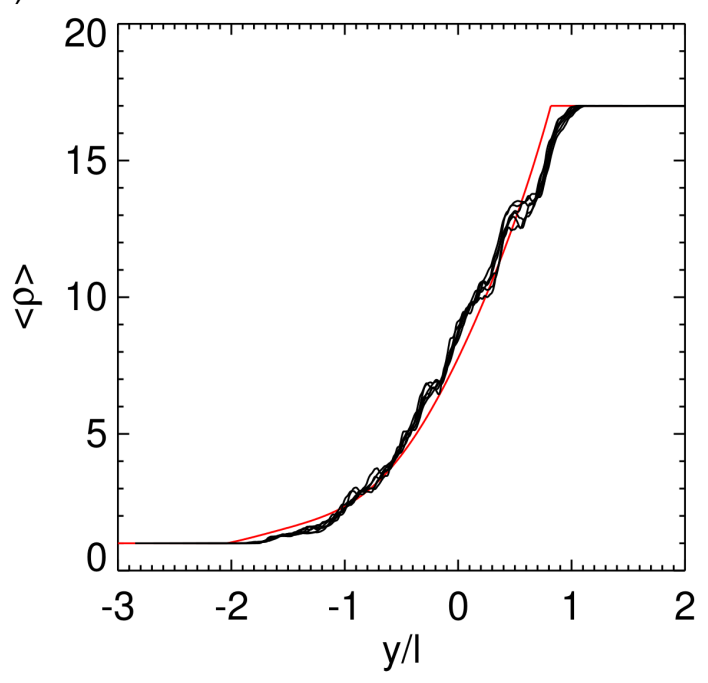

FIG. 10. Simulations of hydrodynamic RT mixing when the density difference is $\Delta \rho / \rho_{1}=0.1$ (a) and $\Delta \rho / \rho_{1}=16$ (b). The panels show the average in the $x$ direction of the density over a wide range of times. The axis has been normalised by the self-similar variable $\alpha_{u} A g t^{2}$ so that the solutions lie on top of each other. The black line in the each of these figures is the mean profile calculated over the different snapshots used. The red line shows the model density distribution.

\section{SUMMARY}

In this paper we have laid out a method to use a hierarchy of self-similar solutions to describe the height, depth, and density distribution of the mixing layer as a result of the Rayleigh-
Taylor instability in an immiscible fluid or a miscible fluid in the small mass diffusion limit. The method applied in this paper should be understood as a form of energy minimisation involving the mean density that allows the energy to be efficiently released based on local arguments for a monotonic 
(a)

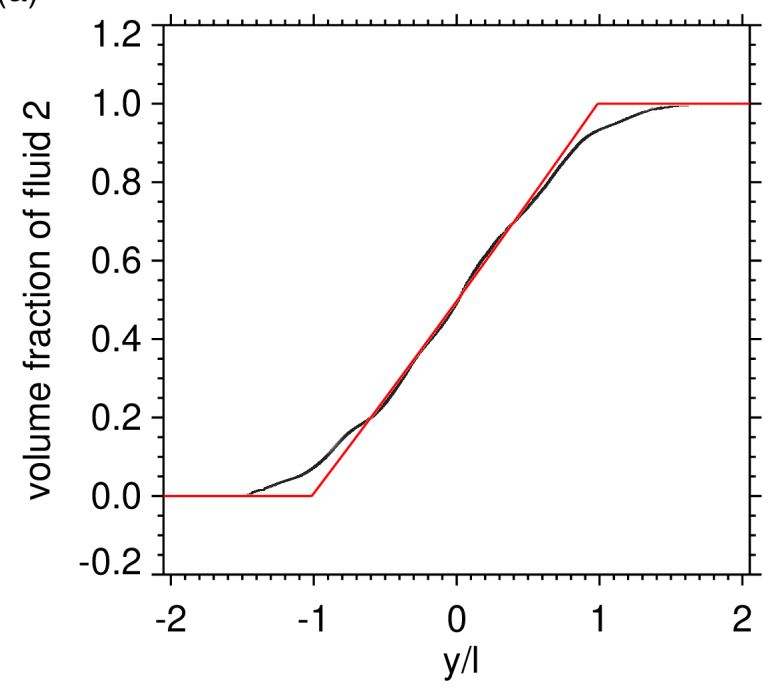

(c)

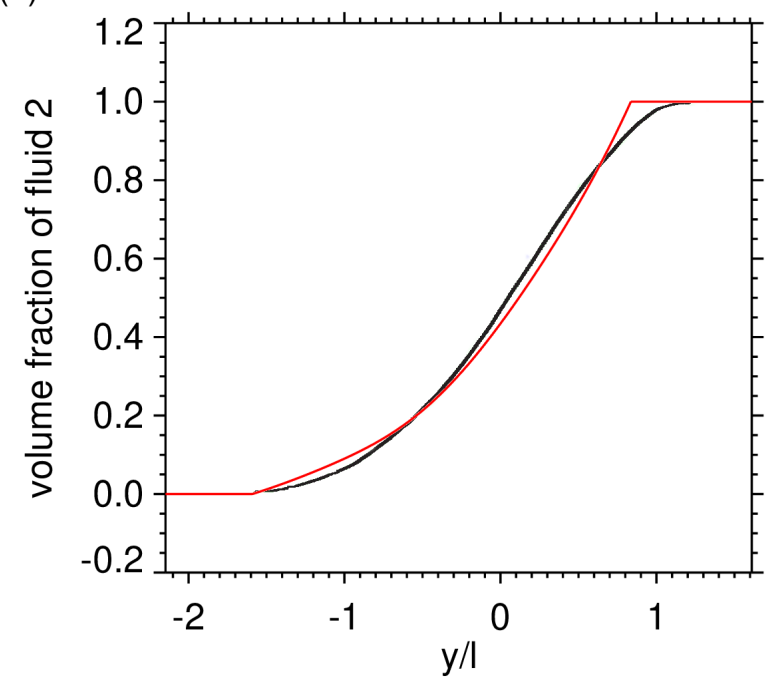

(b)

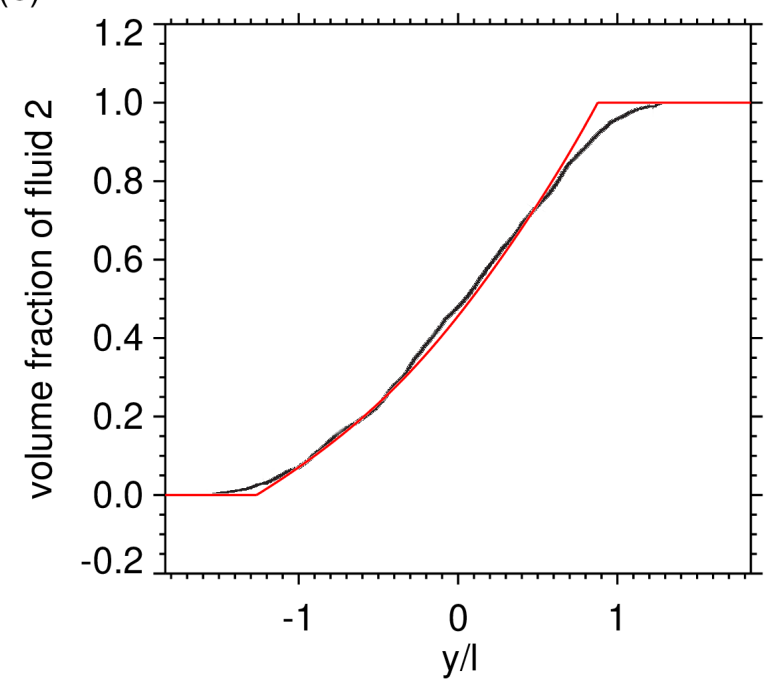

(d)

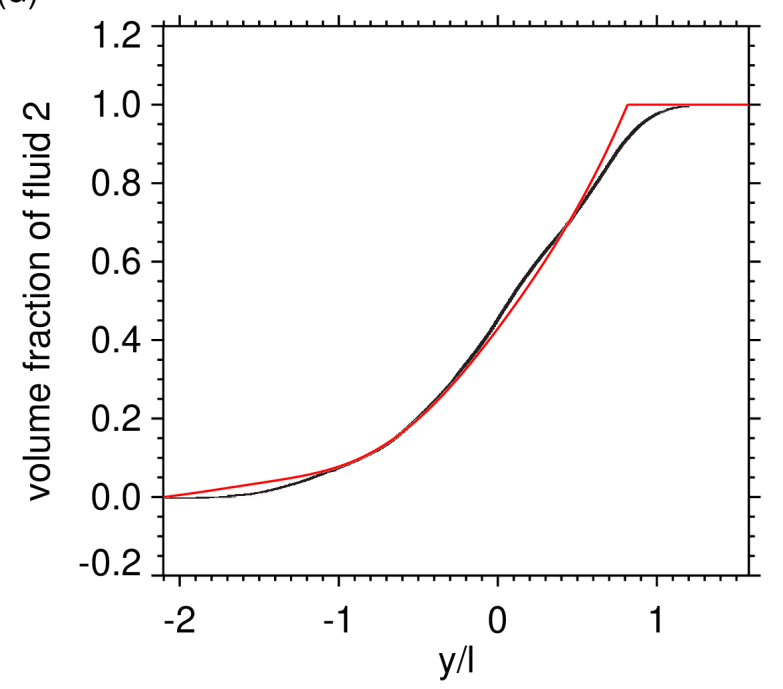

FIG. 11. Comparison between simulation data (solid black line) taken from Livescu, Wei, and Petersen ${ }^{25}$, Journal of Physics: Conference Series 318, 082007 (2011), reproduced with permission, and the model mixing fraction profile (red line) at $A=0.04$ (a), $A=0.5$ (b), $A=0.75$ (c) and $A=0.9$ (d)

density profile.

This model leads to the following predictions:

1. The thickness of the layer has a more complex dependence on the density than just being linearly dependent on the Atwood number (as given in Equation 1), but there is the value, $\alpha_{\mathrm{u}}$, that can describe the properties of the mixing layer that is independent of the density ratio.

2. For very small density differences the density profile becomes approximately linear with a thickness determined by $2 \alpha_{\mathrm{u}} A g t^{2}$.

3. For large density differences the mixing of the lower layer is more efficient that the upper layer resulting in an asymmetric profile of the mixing with a large increase in the measured $\alpha_{\mathrm{s}}$ and a small decrease in $\alpha_{\mathrm{b}}$ as Atwood number increases.

4. A monotonically increasing density distribution in the mixing layer with the $m^{\text {th }}$ derivative of the distribution at either edge of $A^{m} \rho_{1}$ and $A^{m} \rho_{2}$ which can be approximated by a polynomial. This provides a reasonable match to the density distributions found in experiments and numerical simulations.

Beyond understanding the density distribution and energy release of the instability, one application of this model is clearly as a benchmarking method for numerical codes.

An important corollary of this model is that it supports the existence of a mixing parameter, which we call $\alpha_{\mathrm{u}}$, that char- 
(a)

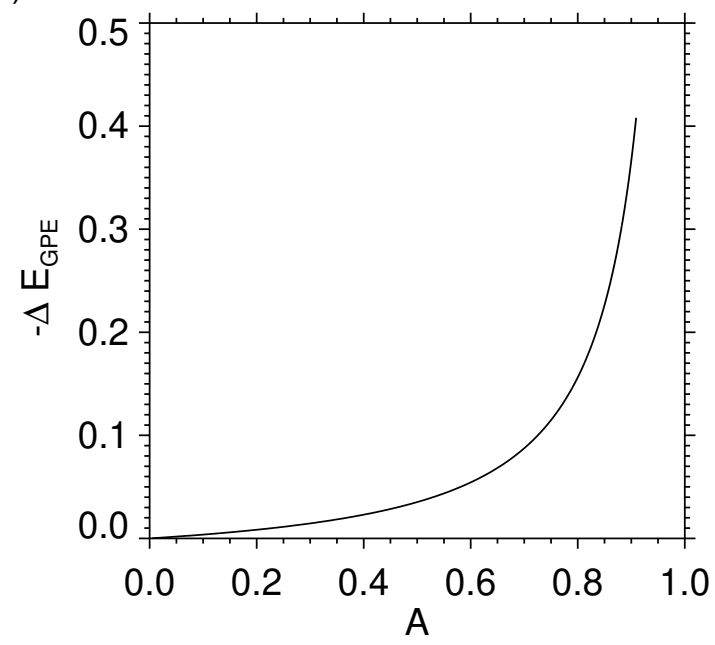

(b)

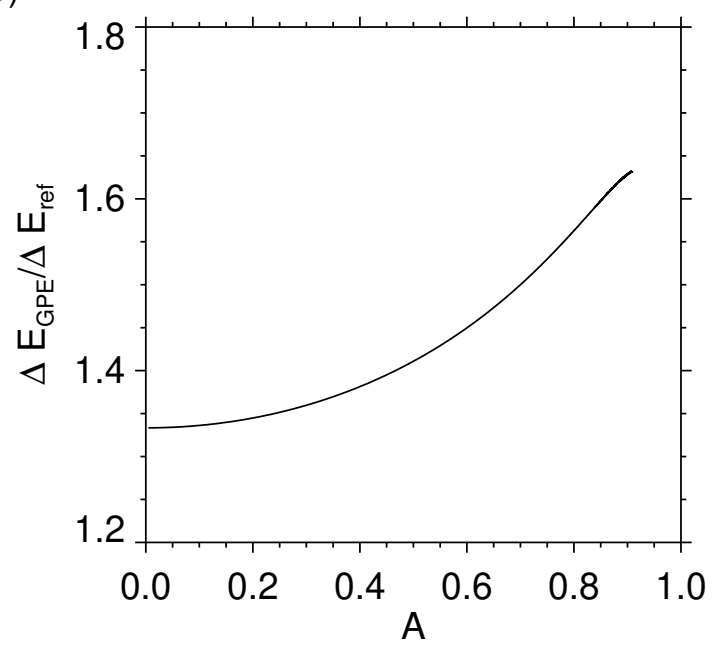

FIG. 12. Plot of the change in energy given by the nonlinear instability against Atwood number (a) and the change normalised by the energy change given by the first step of the expansion against Atwood number (b).

acterises the mixing layer asymmetry as just being a function of the Atwood number. This implies that a suite of related experiments, or numerical simulations, over a large range of Atwood numbers (for example those shown in Figure 3) where the different $\alpha$ values exist for both bubbles and spikes and for different Atwood numbers, a single mixing parameter $\alpha_{u}$ could be determined. This $\alpha_{u}$ is independent of the initial Atwood number though not other important aspects that determine the mixing, e.g. initial flow perturbation or mass diffusion are still crucial in determining $\alpha$ and the asymmetry of the layer ${ }^{9,12,13}$.

As we have previously mentioned, miscible simulations with sufficiently large mass diffusion ${ }^{15,16,20}$ show a different asymmetry of the mixing layer than that found in the model presented here. Changes in the asymmetry of the mixing layer were also found to occur as the result of the inclusion of magnetic fields ${ }^{27}$. One interesting and important development of the model presented here would be to include these effects to see if the asymmetry found in those situations can be reproduced. The comparison with miscible simulations ${ }^{25,26}$ in Section III B 1 highlight that the model can reproduce the general structure of a miscible mixing layer. However, the upper regions of the mixing layer show a consistent expansion of the mixing layer in the simulations over the model. Determining the role of mass diffusion in creating this overshoot could be an important aspect in understanding the smaller asymmetries found in miscible simulations.

In the model presented here, Eqns. 9 and 10 determine the width of the mixing layer of the model. One question is: can this be used in any way to assess the width of the mixing layer found in experiments or simulations? The formulation presented here for determining the width of the mixing layer is inherently determined by the conditions at the middle of the layer. The implication being that a width defined as

$$
W_{\text {mix }}=\frac{\Delta \rho}{2}\left(\frac{d}{d y}(\langle\rho\rangle)\right)^{-1},
$$

where the derivative is taken at $y=0$, would provide an alternative measure of the width. Figure 13 shows the variation of the non-dimensional width calculated from the model using this formulation against Atwood number. Though the formulation does not fully remove the Atwood number dependence in calculating the width, it is reduced to be at the level of a fluctuation of $<10 \%$. To reduce noise affecting this calculation from real data, the derivative would have to be calculated from the derivative of a curve (or straight line) fitted to the data around $y=0$. There are possible connections with this method of measuring the width of the mixing layer and the relation found of the mass flux at the centre of the $y=0$ position of the mixing layer and the growth of the mixing region ${ }^{28}$. However, these are beyond the scope of the current work.

An alternative idea is that (assuming the conditions for this model to be applied are satisfied) the use of Eqn 36 to determine $\alpha$ could be modified. The $h$ and $\dot{h}$ values for a number of values of $\langle\rho\rangle$ could be determined at a given time, and then $\alpha_{\mathrm{u}}$ could then be calculated from these using

$$
\alpha_{\mathrm{u}}=\overline{\frac{\dot{h}(\langle\rho\rangle)^{2}}{4 \operatorname{Agh}(\langle\rho\rangle) L(\langle\rho\rangle)}}
$$

where $L(\langle\rho\rangle)$ the normalisation factor for a given $\langle\rho\rangle$ calculated from the model, and the overline is used here to denote averaging across different $\langle\rho\rangle$ values. As such the $\alpha_{u}$ value that is determined by $h$ and $h$ across a wide range of $\langle\rho\rangle$ could then be averaged together, improving the statistics (and hopefully the accuracy) of this determination of $\alpha$.

The ability to determine the mixed density profile of a the layer allows the energy released by the instability to be calculated. This in turn, by the conservation of energy, gives 


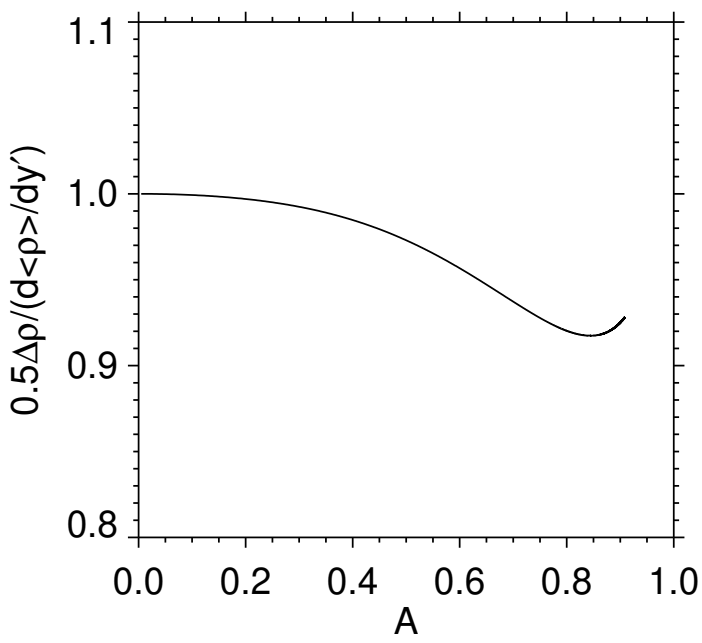

FIG. 13. Measure of the non-dimensional mixing width of the mixing model as calculated using Equation 38 against Atwood number.

an estimate of the kinetic energy in the mixing layer at any given time. This can be used as an upper bound on the turbulent kinetic energy of the layer. Calculating this released energy could be an important addition to 1D models that should include Rayleigh-Taylor mixing, for example those used to model Supernovae.

\section{ACKNOWLEDGMENTS}

Andrew Hillier is supported by his STFC Ernest Rutherford Fellowship grant number ST/L00397X/2 and STFC research grant ST/R000891/1. I would like to thank Dr J. Carlyle, Dr T. Matsumoto, Prof A. Gilbert and Dr. Y. Zhou for their useful discussions. This work used the COSMA Data Centric system at Durham University, operated by the Institute for Computational Cosmology on behalf of the STFC DiRAC HPC Facility (www.dirac.ac.uk). This equipment was funded by a BIS National E-infrastructure capital grant ST/K00042X/1, DiRAC Operations grant ST/K003267/1 and Durham University. DiRAC is part of the UK National E-Infrastructure.

${ }^{1}$ L. Rayleigh, "Investigation of the character of the equilibrium of an incompressible heavy fluid of variable density," Proceedings of the London Mathematical Society 14, 170-177 (1883).

${ }^{2}$ G. I. Taylor, "The Instability of Liquid Surfaces when Accelerated in a Direction Perpendicular to their Planes. I," Proceedings of the Royal Society of London Series A 201, 192-196 (1950).

${ }^{3}$ S. F. Gull, "A numerical model of the structure and evolution of young supernovaremnants," MNRAS 161, 47-69 (1973).

${ }^{4}$ R. D. Petrasso, "Rayleigh's challenge endures," Nature (London) 367, 217 218 (1994).
${ }^{5}$ T. E. Berger, G. Slater, N. Hurlburt, R. Shine, T. Tarbell, A. Title, B. W. Lites, T. J. Okamoto, K. Ichimoto, Y. Katsukawa, T. Magara, Y. Suematsu, and T. Shimizu, "Quiescent Prominence Dynamics Observed with the Hinode Solar Optical Telescope. I. Turbulent Upflow Plumes,” Astrophys. J. 716, 1288-1307 (2010).

${ }^{6}$ A. Hillier, "The magnetic Rayleigh-Taylor instability in solar prominences," Reviews of Modern Plasma Physics 2, 1 (2018).

${ }^{7}$ E. Fermi and J. von Neumann, "Taylor instability of incompressible liquids," U.S. Government Document AECU-2979 (1955).

${ }^{8}$ J. R. Ristorcelli and T. T. Clark, "Rayleigh Taylor turbulence: self-similar analysis and direct numerical simulations," Journal of Fluid Mechanics 507, 213-253 (2004).

${ }^{9}$ Y. Zhou, T. T. Clark, D. S. Clark, S. Gail Glendinning, M. Aaron Skinner, C. M. Huntington, O. A. Hurricane, A. M. Dimits, and B. A. Remington, "Turbulent mixing and transition criteria of flows induced by hydrodynamic instabilities," Physics of Plasmas 26, 080901 (2019).

${ }^{10}$ D. H. Sharp, “An overview of Rayleigh-Taylor instability," Physica D Nonlinear Phenomena 12, 3 (1984)

${ }^{11} \mathrm{G}$. Dimonte et al., "A comparative study of the turbulent Rayleigh-Taylor instability using high-resolution three-dimensional numerical simulations: The Alpha-Group collaboration," Physics of Fluids 16, 1668-1693 (2004).

${ }^{12}$ Y. Zhou, "Rayleigh-Taylor and Richtmyer-Meshkov instability induced flow, turbulence, and mixing. I," Phys. Rep. 720, 1-136 (2017).

${ }^{13}$ Y. Zhou, "Rayleigh-Taylor and Richtmyer-Meshkov instability induced flow, turbulence, and mixing. II," Phys. Rep. 723, 1-160 (2017).

${ }^{14}$ G. Dimonte and M. Schneider, "Density ratio dependence of RayleighTaylor mixing for sustained and impulsive acceleration histories," Physics of Fluids 12, 304-321 (2000).

${ }^{15}$ W. H. Cabot and A. W. Cook, "Reynolds number effects on RayleighTaylor instability with possible implications for type Ia supernovae," Nature Physics 2, 562-568 (2006).

${ }^{16} \mathrm{Y}$. Zhou and W. H. Cabot, "Time-dependent study of anisotropy in Rayleigh-Taylor instability induced turbulent flows with a variety of density ratios," Physics of Fluids 31, 084106 (2019).

${ }^{17}$ G. Dimonte, "Dependence of turbulent Rayleigh-Taylor instability on initial perturbations," Phys. Rev. E 69, 056305 (2004).

${ }^{18}$ A. Banerjee, W. N. Kraft, and M. J. Andrews, "Detailed measurements of a statistically steady Rayleigh-Taylor mixing layer from small to high Atwood numbers," Journal of Fluid Mechanics 659, 127-190 (2010).

${ }^{19}$ G. C. Burton, "Study of ultrahigh Atwood-number Rayleigh-Taylor mixing dynamics using the nonlinear large-eddy simulation method," Physics of Fluids 23, 045106-045106 (2011).

${ }^{20} \mathrm{~W}$. Cabot and Y. Zhou, "Statistical measurements of scaling and anisotropy of turbulent flows induced by Rayleigh-Taylor instability," Physics of Fluids 25, 015107-015107-18 (2013).

${ }^{21}$ A. G. W. Lawrie and S. B. Dalziel, "Rayleigh-Taylor mixing in an otherwise stable stratification," Journal of Fluid Mechanics 688, 507-527 (2011).

${ }^{22}$ A. Hillier, S. Takasao, and N. Nakamura, "The formation and evolution of reconnection-driven, slow-mode shocks in a partially ionised plasma," A\&A 591, A112 (2016).

${ }^{23}$ M. Rempel, M. Schüssler, and M. Knölker, "Radiative Magnetohydrodynamic Simulation of Sunspot Structure," Astrophys. J. 691, 640-649 (2009), arXiv:0808.3294.

${ }^{24}$ J. P. Mellado, S. Sarkar, and Y. Zhou, "Large-eddy simulation of RayleighTaylor turbulence with compressible miscible fluids," Physics of Fluids 17, 076101-076101-20 (2005).

${ }^{25}$ D. Livescu, T. Wei, and M. R. Petersen, "Direct numerical simulations of rayleigh-taylor instability," Journal of Physics: Conference Series 318, 082007 (2011).

${ }^{26} \mathrm{D}$. Livescu, "Numerical simulations of two-fluid turbulent mixing at large density ratios and applications to the Rayleigh-Taylor instability," Philosophical Transactions of the Royal Society of London Series A 371, 20120185-20120185 (2013).

${ }^{27} \mathrm{~J}$. Carlyle and A. Hillier, "The non-linear growth of the magnetic RayleighTaylor instability," A\&A 605, A101 (2017).

${ }^{28}$ A. W. Cook, W. Cabot, and P. L. Miller, "The mixing transition in Rayleigh Taylor instability," Journal of Fluid Mechanics 511, 333-362 (2004). 